\title{
5-OR: Health Disparities in People with and without Diabetes during the COVID-19 Pandemic
}

\author{
1. BARBARA A. MYERS, \\ 2. RACHEL KLINGENSMITH and \\ 3. MARY DE GROOT
}

Diabetes 2021 Jun; 70(Supplement 1): -. https://doi.org/10.2337/db21-5-OR

\begin{abstract}
Purpose: To characterize the psychosocial experiences of adults with (PWD) and without diabetes (ND) during the COVID-19 pandemic. US adults (2176) completed a web-based survey in May-June, 2020 and November-December, 2020, including demographics, COVID-19 exposure, diabetes-related distress (DDS-17), depressive symptoms (PHQ-8) and anxiety (GAD7). At baseline, mean age was 49.6 years (S.D. $=16.9), 80 \%$ female, $88.3 \%$ White, with an annual household income of $\geq \$ 60,000$ (57.6\%), type 2 diabetes (T2D; 301,13.9\%), 145 prediabetes $(145,6.6 \%)$ and type 1 (T1D, 100, 4.6\%). One-third $(29.7 \%)$ reported decreased income due to the pandemic. T2Ds had more medical comorbidities and COVID risk factors than T1Ds and NDs (all $\mathrm{p}<0.01$ ). Mean PHQ-8 scores were 7.1 (S.D. = 5.8; mild), with the T2Ds (M $=7.7$; S.D. $=5.9)$ exceeding NDs $(M=6.9 ;$ S.D. $=5.7 ; \mathrm{p}<.001)$. Mean DDS-17 and GAD-7 scores were comparable for T1Ds and T2Ds (moderate level; $p=N S)$. At 6 months (6MFU), 1,345 (62.6\%) completed follow up surveys. Completers were more likely to be older, male, White, married, with higher education levels, and homeowners, with a greater proportion of medical comorbidities and lower Alcs at baseline than non-completers (all $\mathrm{p}<.05$ ). $6 \mathrm{MFU}$ completers had lower baseline depressive symptoms and diabetes distress, lower household COVID-19 rates and less difficulty paying bills than non-completers (all $\mathrm{p}<.05$ ). At $6 \mathrm{MFU}$ for all groups, depressive symptoms decreased $(\mathrm{p}<.0001)$ and financial strain improved $(\mathrm{p}<.001)$, while COVID exposure increased (personal and household, $\mathrm{p}<.001$ ). Diabetes distress remained at a moderate level for T1Ds and T2Ds. T1Ds and T2Ds showed comparable levels of depressive symptoms to NDs but were more likely to report financial hardship $(\mathrm{p}<.05)$ and difficulty paying bills than NDs $(\mathrm{p}<.001)$.
\end{abstract}

Health outcomes were worse for PWDs compared to NDs during the COVID-19 pandemic despite high SES protective factors. Persistent financial strain and diabetes distress increase the risk for future poor health outcomes.

Disclosure B. A. Myers: None. R. Klingensmith: None. M. De groot: Consultant; Self; Johnson \& Johnson.

This is the author's manuscript of the article published in final edited form as:

Myers, B. A., Klingensmith, R., \& Groot, M. D. (2021). 5-OR: Health Disparities in People with and without Diabetes during the COVID-19 Pandemic. Diabetes, 70(Supplement 1). https://doi.org/10.2337/db21-5-OR 